\title{
NEW INTERESTING EULER SUMS
}

\section{AmRiK Singh Nimbran And AnThony Sofo}

Abstract. We present here some new and interesting Euler sums obtained by means of related integrals and elementary approach. We supplement Euler's general recurrence formula with two general formulas of the form $\sum_{n \geqslant 1} O_{n}^{(m)}\left(\frac{1}{(2 n-1)^{p}}+\frac{1}{(2 n)^{p}}\right)$ and $\sum_{n \geqslant 1} \frac{O_{n}}{(2 n-1)^{p}(2 n+1)^{q}}$, where $O_{n}^{(m)}=$ $\sum_{j=1}^{n} \frac{1}{(2 j-1)^{m}}$. Two formulas for $\zeta(5)$ are also derived.

Mathematics subject classification (2010): 11M06, 11M32, 11Y60, 33B15, 40A25, 40B05.

Keywords and phrases: Euler sums, harmonic numbers, gamma function, psi function, zeta function, polylogarithm function.

\section{REFERENCES}

[1] G. BASTIEN, Elementary methods for evaluating Jordan's sums $\sum_{n \geqslant 1}\left(1+\frac{1}{3}+\cdots+\frac{1}{2 n-1}\right) \frac{1}{n^{2 a}}$ and $\sum_{n \geqslant 1}\left(1+\frac{1}{3}+\cdots+\frac{1}{2 n-1}\right) \frac{1}{(2 n-1)^{2 a}}$ and analogous Euler's type sums and for setting a $\sigma$-sum theorem, 2013, http://arxiv.org/abs/1301.7662v1.

[2] B. C. Berndt, Ramanujan's Notebooks Part 1, Springer-Verlag, New York 1985.

[3] D. Borwein, J. M. Borwein And R. Girgensohn, Explicit Evaluation of Euler Sums, Proceedings of the Edinburgh Mathematical Society 38 (1995), 277-294.

[4] D. BORWEIN AND J. M. BORWEIN, On an intriguing integral and some series related to $\zeta(4)$, Proc. Amer. Math. Soc. 123 (1995), 1191-1198.

[5] D. Castellanos, The Ubiquitous $\pi$, Math. Mag. Part I, 61(2) 1988, 67-98; Part II, 61(3) 1988, $148-163$.

[6] P. J. De Doelder, On some series containing $\psi(x)-\psi(y)$ and $(\psi(x)-\psi(y))^{2}$ for certain values of $x$ and $y$, J. Comput. Appl. Math. 37 (1991), 125-141.

[7] L. EulER, Meditationes circa singulare serierum genus, Novi Commentarii academiae scientiarum Petropolitanae 20, 1776, 140-186, E477.

[8] P. Flajolet And B. SAlvy, Euler Sums and Contour Integral Representations, Experimental Mathematics, Vol. 7 (1998), No. 1, 15-35.

[9] C. Georghiou And A. N. Philippou, Harmonic Sums and the Zeta Function, Fibo. Quart. 21 (Feb. 1983) 29-36.

[10] P. F. Jordan, Infinite Sums of Psi Functions, Bulletin of the American Mathematical Society, Vol. 79, No.4, July 1973, 681-683.

[11] N. NieLsen, Handbuch der Theorie der Gammafunktion, Leipzig, Druck und Verlag von G. B. Teubner, 1906 (reprinted by Chelsea Publishing Company, Bronx, New York, 1965).

[12] S. Ramanujan, Manuscript Book 2 of Srinivasa Ramanujan, Tata Institute of Fundamental Research, http://www.math.tifr.res.in/ publ/nsrBook2.pdf.

[13] G. Rutledge and R. D. Douglass, Table of Definite Integrals, Amer. Math. Monthly, Vol. 45, No. 8 (Oct., 1938), pp. 525-530.

[14] H. F. Sandham, Advanced Problem 4305, Amer. Math. Monthly, Vol. 55 (7) Aug.-Sep., 1948, p.431; solution by Martin Kneser 57(4) Apr. 1950, 267-268.

[15] R. Sitaramachandrarao, A Formula of S. Ramanujan, Journal of Number Theory 25 (1987), $1-19$. 
[16] A. Sofo And D. Cvijović, Extensions of Euler Harmonic Sums, Appl. Anal. Discrete Math. 6 (2012), 317-328, available online at http://pefmath.etf.rs.

[17] A. Sofo, General order Euler sums with rational argument, Integral Transforms Spec. Funct. (2019), https://doi.org/10.1080/10652469.2019.1643851.

[18] A. Sofo, New classes of harmonic number identities, J. Integer Seq. 2012; 15: no. 7, Article 12.7.4, $12 \mathrm{pp}$.

[19] A. Sofo, Evaluation of integrals with hypergeometric and logarithmic functions, Open Math. 16 (2018), no. 1, 63-74.

[20] A. Sofo, H. M. SRivastava, A family of shifted harmonic sums, Ramanujan J. 37 (2015), no. 1, $89-108$.

[21] G. T. Williams, A new method of evaluating $\zeta(2 n)$, Amer. Math. Monthly 60(1) Jan. 1953, 19-25. 